\title{
RELAÇÃO DAS OBRAS ENTRADAS NA BIBLIO- TECA DA FACULDADE DURANTE O PERIODO COMPREENDIDO ENTRE 16 DE AGOSTO E 15 DE NOVEMBRO DE 1934
}

\author{
OBRAS GERAIS- $(0)$
}

Relatorios, Revistas, Dicionarios, etc.

Anuario da Escola Politecnica-S. Paulo, 1934-1 vol. Permuta.

Anuario da Faculdade de Direito da Universidade de São Paulo-São Paulo, 1934, 1 vol.-Doasäo.

Archives de Philosophie du Droit et de Sociologie Juridique-lParis, 1934 -1 vol.-Compra.

Boletim do Instituto de EngenhariaVol. XIV-Sãd Paulo, 1934-1 vol. Permuta.

Boletin de la Universidad de la Plata -Cultura general y artistica-La Plata, $1922-1924-1$ vol. Permuta.

Le Crapouiłiat-Paruis, $1932-1933-2$ vols. Doação.

A Capital de São Paulo em 1933-Sãu Paulo, 1934-1 vol. Doação.

Estatutos da Ordem dos Advogados do Rio Grande do Sul-Porto Alegre, 1927-1 vol. Permuta.

Exposição de Obras Juridicas de Autores Rio Grandenses-Instituto da Ordem dos IAdvogados do Rio Grande do Sul-Porto Alegre, 193-1 vol. Permuta.

Guia Fiscal一São Paulo, 1926-a-1932 -5 volumes.-Doação.

Giorgio del Vecchio-Ricurdando Alberioo Gentile-Ryoma, 1934-1 vol. - Permuta.
Indicador Alfabetico dos Atos Oficiais Gerais Referentes ao Ministerio da Guerra-Rio de Janeiro, 1934-1 vol. -Doação.

Le Mois-Synthèse de Pactivité Mondiale-Juillet-Août; Setembre-Octobre; Octobre - Novembre-Paris, 1934-4 volumes. -Compra.

Pandiá Calogeras na Opinião de seus Contemporaneos-São Paulo, 1934-1 voll.-Doação.

Primeiro Congresso Juridico Rio Grandenso-Comemoração do Centenario da revoluçāo Farroupilha-porto Ale gre, 1933-1 val.-Permula.

Regimento Interno da Faculdade de Ciencias Economicas do Rio de Janeiro-Rio de Janeirs, 1931-1 vol Doação.

Revista de Comercio e Industria-Sāo Paulo, 1915—1921-7 vols.-Doacão.

Revista do Departamento Nacional do Café-Rio de Janeiro, 1934 1vol.Permuta.

Revista de Derecho Penal-Bucnos tires, 1924-1 vol.-Permuta.

Revista do Instituto Geografico e Ilistorico da Baia-N. ${ }^{\circ}$ s 53-a-55-Baia, $1927-a-1929-3$ vols.-Permuta.

Revista de Jurisprudencia BrasileiraVol. XXLLL $\longrightarrow$ Rio de Janeiro, 193セ 1 vol-Permuta.

Revista Nacional-Monsario de intercambio cu!tural no Brasil-Rio de Janeiro, $193+-2$ vols.-Permuta. 
Revista Paulista de Contabilidade - Sáo Paulo, 1922-1933-10 vols.-Doação.

Revista dos Tribunais-Vols. 89 e $90-$ São Paulo, 1934-Permuta.

Revue Trimestrielle de Droit CivilTables Générales des 25 premičres années 1902-1926-Paris, 1929 e 1934 -2 vols.-Compra.

Reynaldo Porchat-Homenagem ao poeta Vicente de Carvalho (Discurso)São Paulo, 1918 -1 vol.-Doação.

\section{FILOSOFIA-(1)}

\section{Filosofia, Psicologia, Espiritismo, Moral, etc.}

Blondel (Maurice-) - La Pensée-Paris, 1934-1 vol.-Compra.

Bougeois (Léon-)-Essai d'une philosophie de la solidarité-Paris.19021 vol.-Permuta.

Codigo de Etica Profissional-Porto Alegre, 1827-1 vol.-Permute.

Jaspers (Ludgero-)-Manual de Filosofia-São Paulo-Rio, 1932-1 vol-Compry.

Levy-Bruhl-La Morale et la Science des Moeurs-Paris, 1927-1 volCompra.

Naville (Ernest-)-La définition de la Philosorhie-Peris, 1894-1 vol.Permita.

Patrascoin (J.-)-Lógica-Buenos Aires, 1932-1 vol.-Compra.

Sighele (Scipio-)-Psycholngie des Sectes-Paris,1898-1 val-Permuta.

\section{CIENCIAS JURIDICAS E SOCIAIS-(3)}

Sociologia, Politica, Estatistica, Eco. nomia, Direito, Medicina Legal, Educação, Costumes. etc.

A. Ferreira Coelho-Codigo Civil das Estados Unidos do Brasil-Vol, XXVI -Rio de Janeiro, 1933-1 vol.-Compra.

Affonso de Toledo Bandeira de Mello-politica comeroial do Brasil-Rio de Janeira, 1933-1 vol.-Doação.
Alcino Bittencourt de Abreu-Contribuicão ao estudo medico-legal das manchas de leite e colostro-S. Paulo, 1928-1 vol.-Doação.

Almiro dos Reis-A mordedura na identificação-São Paulo 1927-1 vol. $\rightarrow$ Dokação.

Almiro dos Reis-A dentada na identificação-São Paulo, 1926-1 vol.Doação.

Alvaro Pedro dos Santos-Contribuição ao estudo de revisão dos cristais de hemocromogenio-São Paulo, 1928 -1 vol.-Doação.

Anchorena (Bonito A. Nazar-)-Discurso pronunciado el dia $10^{\circ}$ de deciembre de 1917, en el acto de la transmision de la Presidencia de la Iniversitad-La Plata, 1927-1 vol. 一Permuta.

Anchorena (Benito A. Nazar-)Acusación fiscal em el proceso por defraudación de las marcas de Secrestat $\mathrm{y}$ de Bardinet-Buenos Aires, -1914-1 vol.--Permuta.

Anchorena (Benito A. Nazar-)-Derecho de los legisladores electos para ejercer su mandato mientras no se rechase el diploma que les acredita-Bunos Aires, 1928-1 vol.-Permuta.

Anchorena (Benito A. Nazar-)-Discurso inaugural $y$ plan de estudios de la Facultad de Ciencias Juridicas y Sociales de la Universidad del Litoral--Buenos Aires, $1920 \longrightarrow 1$ vol.Permuta.

Anchorena (Benito A. Nazar-) -Intervencion Nacional en la Provincia de Tucuman.-Buenos Aires, 1922-1 vol. -Doação.

Anchorena (Benito A. Nazar-)-Discurso pronunciado en la sesión plenaria inaugural del Tercer Congreso Universitario anual.-Cordoba. 1926-1 vol.-Permuta.

Añorbes (Juan-)-Reclamacion de la Republica de Pamamá-Panamá, 1934 $\longrightarrow$ vol. $\longrightarrow$ Permuta.

Antonio Dacio Franco Amaral-Experiencias sobre a resistenci do mercurio e do iodo á incineração.-São Pauio, :1930—1 vol.—Doração. 
Areco (Horacio P.-)-El duelo y los fueros parlamentarias-incidente Moreno-Nazar Anchorena-Buenos $\mathrm{Ai}$ res, $1919 \longrightarrow 1$ vol.-Permuta.

Arnaldo de Oliveira Bacellar-A surdo-mudez no Brasil-São Paulo, 1926 -1 vol.-Doacãa.

Ascoli (Max) - La Interpretazione delle Leggi-Roma, 19.28-1 vol-Compra.

Atti del Primo Convenio di Studi Sindicali e Corporativi-Roma, 1930, 1932 -3 vols.-Doação.

Atti del Secondo Convenio di Stadi Sindicali e Corporativi-Roma, 1932 -2 vols.-Doacãa.o.

Attilio Oglietti-Contribuição aro estudo medico-legal das cicatrizes.São Paulo, 1926—1 vol.-Doação.

Aureliano Borges de Carvalho-Vocacão medioa-São Paulo, 1931-1 vol. $\rightarrow$ Doação.

Barthélemy (Joseph-) -Essai sur le Travail Parlamentaire et le systeme des commissions-Paris, 1934-1 vol. -Compra.

Benjamin Credidio-Contribuição para - estudo medico-legal da escoriação -São PPaulo, 1930-1 vol-Doação.

Bento Lacerda de Oliveira- $O$ problema da eutanasia-São Paulo, 1925-1 vol.—Doação.

Borgoño (Luis Barros-)-Don Jua Maria Gutićrrez-Atraves de una correspondencia-Santiago, 1934-1 vol.-Permuta.

Bonnard (Roger-)-Le controle juricictionnel de l'administration-Paris, 1934 - 1 vol...-Сompra.

Bourgeois (Pierre-)-Les transports urbains du grand Paris-Paris,19271 val.-Permuta.

Brandino Francisco Genovesi-O peso e as dimensões do féto a termo para fins medico-legais-São Paulo, 1928 -1 vol.-Doação.

Brasil e Japão-São Paulo, 1934-1 vol.-Donção.

Carlos de Campos Pagliuchi-A cirurgia estetioa sob o ponto de vista moral e legal-São Paulo, 1931-1 vol. $\rightarrow$ Donacáo.
Carlos Noce-Intoxicaçoes euforicas e sua profilaxia-São Paulo, 1926-1 vol.-Doação.

Caselli (Abundio-)-Comision genera] de redamaciones entre Panamá y Estadns Unidos da América-Panamá, 1:33-1 vol.-Doação.

Catalano (E.-)-La riforma penale e i suoi riflessi educative-Palermo, 1930—1 vol.—Doação.

Catellani (E. L.-) -Il Diritto Internazionale Privato-Torino, 1895-2 vols.-Permuta.

Chase (William Gerald-)-Comisiun gencral de reclamaciones entre $\mathrm{Pa}$ namá y Estadiks Unidos de América -Panamá, 1934-1 vol.-Permuta.

Charte du Travail (La)-Roma, 19331 vol.-Doação.

Cid Cordeiro Prestes-Ligeira contribuição ao estudo medico-legal das equimoses.-Sāo Paulo, 1922-1 vol.

Ciência do Direito-Revista dos Juizes * Juristas brasileiros-Tomos II e LI -Rio de Janeiro, 1934-1 vol.Compra.

Coleção das Leis c Decretos do Estado de São Paulo-Tomo XLIV.--São Pauln, 193t—1 vol.-Doasão.

Colunje (Guilhermo-)-Comision general de reclamaciones entre Panamá y Estados Unidos de América1 vol. -Permuta.

Comercio de Cabotagem do BrasilAnos de 1931-1932 -1933—Rin de Ji2neiro, 19R4-1 vol.-Daação.

Constantino Catalanom A ação dos projetis de arma de fogo sobre as vestes-São Paulo, 1928-1 vol.-Doacão.

Cunha Bueno Junior-Filiação fraudulenta-São Paulo, 1934-1 vol.-Doasão.

Dario Augasto de Carvalho FrancoContribuição e identificação dos alcatoides por provas microquimicas -São Paulu, 1928-1 vol.-Draçāo.

Davy (Geurges-)-Sociologues d'hier et d'aujourd'hui-Paris, 1931-1 vol. - Compra.

Del Vecchio (Giorgio-) - La crisi della scienza del Diritto-Moderna, 1933.1 vol-Permuta. 
Del Vecchio (Griorgio-)-Individuo, Stato e Conporazione-Roma, 19341 vol.-Permuta.

Del Vecchio (Giorgio-) -Stato fascista e vecchio regime contro il medievalismo giuridico-Roma, 1932-1 vol. $\rightarrow$ Permuta.

Del Vecchio (Gi,orgio-) -ll problema della fonti del diritto positivo-Roma, 1934-1 vol.-Permuta.

Demetrio de Toledo-Vierdade noviRio de Janeiro, 1927-1 vol.-Permuta.

Developpement et L'Activité de L'Organisation Corporative Pendant les dix Premiéres Années de L'Ecre Fasciste (Le)-Roma, 1933-1 vol. $\rightarrow$ Doação.

Diaz (José Maria Vasquez-)-Comision General de Reclamaciones entre Panamá y Estados Unidos de América-Panamá, 1934—1 vol.-Permuta.

Dirceu Vieira dos Santos-O direito de curar-Estudo de jurisprudencia médica-São Pallo, 1924-1 vol.Doação.

Discours du Duce sur la Constitution des Corporations-Roma, 1933-1 vol. $\longrightarrow$ Doação.

Didimo Agapito da Veiga-Do penhor - Manual do Codigo Civil Brasileiro-Vol. IX - (parte quarta) - Rio de Janeiro, 1934-1 vol.-Compra.

Les Doctrines Monétaires a L'Epreuve des Faits-Paris, 193:-1 vol.-Permuta.

Droz (Joseph-)-Economie Politique -Paris, 1854-1 vol.-Permuta.

Durkheime (Émile-)-Le SocialismeParis, 1928-1 vol.-Compra.

Durkheime (Émile-)-Le SuicideParis-1 vol.-Compra.

Eduardo Augusto de Oliveira PirajáAs experiencias "In Anima Nobili" -São Paulo, 1925—1 vol.-Doação.

Edwin (Frederico Zink-)-Docimasia hepatica quimica-São Paulo, 19221 vol.-Droaşão.

El Problema Médico y la Asistencia Médica Mutualistica en Cuba-Habana, 1934-1 vol.-Permuta.

Fistevam José de Almeida Prado-Revișio das reaçôes de Van Deen,
Schaer e de Fleig em Hematologia forense-São Paulo, 1928-1 vol.Doação.

Encario Novaes-Estudo medico legal das manchas de saliva $\longrightarrow$ Săo Paulo, $1920 \longrightarrow 1$ vul. -Doação.

Faustino Ferreira Gomes-Contribuição parra o estudo da resistencia do chumbo á cremação-São Paulo, 1930-1 viol.-Dơação.

Pclicio Laurito-Contribuição ao estudo das manchas de urinas-Vulor do método e da reação do Xanthydrol-São Paulo, 1925-1 vol.-Doação.

Fernando Osorio-A propriedade comercial a o negime das luvas-Rio de Janeiro, 1933-1 vol.-Permuta.

Flaminio Favero-Registo do tipo sanguineo nas cadernetas de identidade -São Paulo, 1934-1 vol.—Doação.

Floriano de Alencar-O Suicidio em $\mathrm{S}$. Paulo-São Paulo, :1926-1 vol.Doação.

Francisco Alves Corrêa de ToledoContribuição ao estudo das tatuagens em medicina legal-São Paulo, 1926 1 vol.-Doksão.

Francisco Lamanéres d'Oliveira-Modificações cadavericas das lesões culaneas por instrumento perfurante c perfuro-cortante-São Paulo, 19211 vol.-Dołação.

Francisco Ourique-Contribuição para o estudo da resistencia do cobre do zinco á cremagãa-São Paulo, 1930 -1 vol. -Doação.

Francisco Schlittler-Contribuição ao estudo do tempo de elliminação do meconio-Sào IPaulo, 1927-1 vol.Doação.

Gastāo Fleury Silveira-Determinação da data do uso de uma arma de fogo e da munição-Săo Paulo, 1926-1 vol.-Doação.

Giddings (Franklin H.-)-Principes de sociologie-Paris, 1897-1 vol-Permuta.

Golovine (IVan-)-Siciences de la Politique-Paris, 1844 1 vol.-Permuta.

Gusmano Oswaldo C. Rinaldo-Vida média provavel nós, e o valor me- 
dico begal.-São Paulo, 1930 -1 vol. -Doação.

Han (Lin-)-Reforme de l'education contemparadine en Chine-Paris, 1933 -1 vol.-Permuta.

Hegel (Giorgio Guglielmo Federico-) -Lineamenti di Filosofia del Dirit. to-Bari, 1913-1 vol.-Permuta.

Hilario Veiga de Carvalho-Contribuicão para o estudo da epimicrascopia em medicina legal-São Paulo, 1929 -1 vol.—Doação.

Honorato Faustino de Oliveira Junior -Lesões por arma de fogo-São Paulo-1 vol. Doašão.

Intervención Judicial en las Sociedades Anonimas-Buenos Aires, 19341 vol.- Piermuta.

IsIas (Guilkermo Garbarine-)-Derecho Rurad Argentino-Buenos Aires, 1925-1 vol.-Doação.

J. Gomes dos Reis Junior-A lavagem colorimetrica do figado, pelo metodo de Chavigny, na diagnose da morte por dessangramento-São Pauro, 1923-1 val.-Doasão.

João Carlos Gomes Cardim-Da prova sulfidrica de Jeard (Contribuição para o seu estudo)—São Paulo, 1929 -1 vol.—Doação.

João Octavio Nebias-Dos estigmas profissionaus-São Paulo, 1926-1 vol. -Doaçãn.

João de Souza Dias-Da pericia medico-begal na electroplesão-São Paulo, 1927-1 vol..-Doaçäro.

Joaquim Caetano da Silva-L'Oyapoc et l'Amazone: question brósilienue et française-Tome I et II-Paris, 1861-1 vol.-. Permuta.

Jorge Hurley- No dominio das aguas —Pará, 1933 —1 vol.—Doacão.

Jorge Tibiriçä Filho- $\mathrm{Da}$ diagnase da distancia nos tiros de projeteis multiplos (chumbo de caça) —São Paulo, $1921-1$ vol.—Doação.

José Anderson-Contribuição para o estudo medicolegal da reação de Bokarins-São Plaulo, 1932-1 vol.Doação.

José Forster Junior-Tecnica da docimasia pararenal-São Paulo, 1921-1 vol. -Daação.
José Guilherme Whitaker-A questão do trabalho de menores en fabricas em São Paulo-Sāo Paulo, 1932-1 vol. $\rightarrow$ Doação.

José Pereira da Silva-Novos rumos da crimionologia-Rio de Janeiro, 19341 vol.-Compra.

José Riberiro Escobar-A construçâo ciêntifica dos programas-1." parteSāo Paulo, 1934-1 vol.—Doaçāo.

José Silveira-Contribuição para o estudo da identificacão especifjea do 'sangue palas catalasos-São Paulo, 1931—1 vol.-Daação.

José Torres de Rezende-Contribuição para o estudo medico-legal da subsstancia fecal-São Paulo, 1929-1 vol. -Doação.

Julio dos Reis Filho-Revisão das retçōes de Meyer e de Soler em homitologia forense-São Paulo, 1927-1 vol.-Doação.

Kantorovicz (Herman U.-) - La holla per la scienza del diritto-Milano, 1908-1 rol.—Doação.

Laurenio Lago-O Estado Maior General do Exeroito Brasileiro-Rio de Janeiro, 1934 - 1 vol.—Doaçāo.

Lee (Richard Joseph-)-Comision general de reclamaciones entre Panamá y Estados L'nudos de AmóricuParamá, 19:34-1 vol.-Doação.

Leonidio Ribeiro-Policia Cientific:Rio de Janusiro, 193ł 1 vol-Compra.

Lettic (Charlot Denham) y Frank Parlin Denham-Comision general de reclamaciones entre Panamá y Estados Ĺnidos de América-Panamá, 1934-1 vol.—Doação.

Levi (Maria Attilio-)-La Constituzione Romana dai Gracchi e Giulio Cesare-Fironze, 1928-1 rol-Compra.

L. F. da Camara Leal-O imposto de transmisaão-Rio de Janeiro, 1871-1 vol.—Doação.

L. Nogueira Paula-Teoria racional dos sistemas economicos-Rio de Janeiro, 1932-1 vol.-Doação.

Luiz de Assis Pacheco Borba-Contribuiçāo para o estudo da identificacão quimica das polvoras queimadas -São Paulo, 1929-1 vol,-Doaçāo.

Luiz Celestino Pereira Ramos-A deon- 
tologin medica e o feticidio medicoSão Paulo, 1920-1 vol.-Doação.

Luiz Ferraz de Sampaio-Da cronologia om tanatologia forense- São Paulo, 1929-1 vol. $\rightarrow$ Doação.

Luiz Gonzaga Melillo-O valor da prova testemunhal-São Paulo, 1926-1 vol.-Doaşāo.

Luiz de Sampaio Arrada-0 diagnostico da epilepsia pela prova da iperpnea em seu aspecto deontologicoSão Paulo, 1930-1 vol.—Doação.

Luiz Splendore-O ponto de Déclard e seu valor medico-legal-São Paulo, 1928-1 vol.-Doaşão.

Manoel de Abreu-A docimasia pulmonar histologica-São Paulo, 1928-1 vol.-Doasãào.

Maranini (Giuseppe-)-Le Origine dello Statuto Albertino-Firenze, 1926 -1 vol.-Compra.

Marcello Guimarães Leite-Contribuisão ao estudo das polvoras e modo de as identificar-São Paulo, 19251 vol.-Doação.

Mariano Leonel Netto-Do estado anterior nos infortunios de trabalhoSão Paulo, 1926-1 vol.-Doação.

Mario Uzzo-Inconveniencias do sistema brasileiro de habilitação dos medicos extrangeiros - São Paulo, 19291 vol.-Doação.

Martner (Daniel-)-Economia Polica-Santiago do 'Chile, 1934-1 vol.Permuta.

Matta (J. Caeiro da-)-Le différend Luso-Allemand sownis au Tribuna] Arbitra! D'Interpretation de ParisLisbonne, 1934 1 vol. -Permuta.

Mauricio Ciubé de Oliveira-Contribuicão para o estudo da sôro-precipitasão em medicina legal-São Paulo, 1931-1:ol_-Doação.

Mersch (Emile-)-Sociogênese da pampa brasileira-Pelotas. 1927-1 vol. -Permuka.

Messias Teixeira Camargo Filho-Contribuições psicologicas que influem nos accidentes de trabalho-São Paulo, 1922-1 vol.-Doação.

Michelis (Giuseppe-) - La oorporazione nel mando-Milano, $1934-1$ vol.Compra.
Miguel Reale-O estado moderno-Rio de Janciro, 1934-1 vol.--Doação.

Moacyr Silveira da Cunha-Cronolosia dos fenomenos cadavericos em São Paulo-São Paulo, 19:25-1 vol.-Doação.

Mucio Drumond Murgel-Contribuição para o estudo da docimasia hepatica histologica-São Paulo, 1929-1 vol. -Doasaão.

Murray (Robert A.-)-Le nozione deldo Stato, dei bisogni pubblici e dell'Attività finanziaria-Roma, 1913-1 vol. -Compra.

Nicolau Sarno-Determinação da idade no vivo pela radiografia dos ossos das extremidades-São Paulo, 19261 vol.-Doação.

Organisation Syndicale et Corporative Italienne (L')-Roma. 1933-1 vol. -Doação.

Olagnier (Paul-)-Le Droit D'Autcur Tome I-e-rI-Paris, 1934-2 vols.Compra.

Palacios (Alfredo L-) - El socialismo argentino y las reformas penalesBuenos Aires, 1934-1 vol.-Doação.

Paulo Sohn-Sobre as lesóes conporais no conccito do Codigo Pienal Brasileiro.-São Paulo, 192:1-1 vol.-Doaç̃̃o.

Paz (Henrique Martinez-) -Introduccion al estudio del derecho civil comparado-Cordoba, 1934-1 vol.Permula.

Pedro Aletto- Contribuiçào para o estudo do diagnosticin da morte real pelo emprego de azul de metilenoSão Paulo, 1930-1 vol.-Doação.

Pedemonte (Grotardo C.-)-Regimen fiscal de seguros-Buenos Aires, 1933 -1 vol-Permuta.

Plinio Martins Rodrigues-Toxicomanias- Meios para combatel-as-São Paulo, 1931-1 vol.-Doação.

Pollitz (Paul-)-Pisicologia do criminuso-Rio de Janciro, 1934 - 1 vol.Compra.

Perels (F.-)-Manuel de Droit Maritime International-Paris, $1884-1$ Vol-Permuta.

Pour la Constitution des Corporations -Diseurs-Rome, 1924-1 vol-Donçān. 
Prius (Adolphe-)-L'Organisation de la liberté et le devoir social.- $\mathrm{Bru}$ xelles-Paris, 189j-1 vol--Permuta.

Programa de Economia Politica-Rio do Jameilo, 1933-1 vol.-Doação.

Ranulpho Pinheiro Lima-A representaça profissional no Brasil-Rio de Janeiro, 1934-1 vol.-Doação.

Rauter (M.-)-Cour's de Procédure Givile Francaise-Paris, 1834-1 vol. -Permuta.

Reybaud (Charles-) -Le Brésil-Paris, 1856-1 vol.-Permuta.

Ricci (Umberto-)-Lezioni de Eoonomia IPolitica-Roma, 1827-1928-1 vol.-Permuta.

Ruiz (José Azacl-)-Comision General de Reclamacionies entre Panamá y Estados Unidos de América-Panamá, 1934—1 vol.—Doação.

Sadalla Amim Ghanem-O Libano (conferencia)-Curitiba,1934- 1 vol.Doação.

Sebastião de Paes e Alcantara-Contribuição ao estudio da tanatognose -Processo do azul de BromotimólSão Paulo, 19:29-1 vol.—Doação.

Stahl (Frédérico Jules-)-Histoire de lat Philosophie du Droit-Paris, 1880 -1 vol.- Permuta.

Sylvio Ognibene-Qontribuição á determinação da idade, no vivo, pela estatura o peso-São Paulo, 1928-1 vol.-Doação.

Sylvio de Godoy Crames-A reação do tribumeto de ouro e o seu valor medico-legal na diagnose do aspermaSão Paulo, 1930 1 vol.-Doação.

Sylvio Varella Martins-Contribuição para o estudio dos cristais de oxihemoglobina cm medicina legal-São Pauo, 1929-1 vol:- $\rightarrow$ Doaçāo.

Tanon (L.-)-L'Evolutian du Droit et la conscience sociale - Paris, 1900-1 vol.-Permuta.

Terencio de Miita-De uma nova teonica na identificação de sangueSão Paulu, 1926-1 vol.-Draşão.

Tito Prates da Fonseca-SociologiaSão Paulo, 1934-1 vol.-Compra.

Valensin (Albert-)-Truité de Droit Naturel-Tome I-et-l-I-Paris, 1925 2 vols.-Compra.
Vicente Marcilio-A doutrina do risco profissional extensivo a profissão do mediqo-São Paulo, 1929-1 vol.Daação.

Vieente Ráo-Direito de Familia dos Soviets-São Paulo, 1932-1 vol.Peimuta.

Vicente Roco Antonio Brescia-O exame de escarro sob o punto de vista medico legal-São Paulo, 1929-1 vol. -Doação.

Vicente Zamitti Mammana-Reação de Barberio (contribuição ao seu estudo)-Sāo Paulo, 1928-1 rol.-Dnação.

Worms (René-)-Organisme e Societé -Paris, 1896-1 vol-Permuta.

Zamora (Niceto Alcalá-)-Le pouvoir juridique sur ce quil est au dela de la vie PFaris, 1934-1 vol.-Compra.

\section{CIENCLAS PURAS-(5)}

Matematicas, fisica, quimica biologia, antropologia, etc.

Dreyfus (Andin'-)-Vida c I'niverso e outros ensaios-São Paulo, 1934-1 vol.-Compra.

Rostand (Jean-)-Les problémes de l'húrédité et du sexe- Paris, 1933-1 vol. -Compra.

\section{CIENCIAS APLICADAS- $(6)$}

Medicina, Engenharia, Comércio, etc.

Adamastor Cortez-Centros de Saucle de São Paulo-São Pauio, 1926-1 vol.—Doação.

Alberto Caldarelli-O clinico e a saúcle publica-São Paulu, 1927-1 vol. $\rightarrow$ Doæação.

Alvaro Augusto de Carvalho FrancoA posição do escolar-t fundação do mobiliario-São Paulo. 1923-1 vl.Doaşāo.

Angelo Pereira de Queiroz-Contrihuição ao estudo da Lepra no BrasilSão Paulo, 1928-1 vol.-Doação.

Antonio de Barros Ulhôa Cintra-Sobre a imunidade natural á Diphteria -Rio de Janciro, 1931-1 vol.-Draรйo. 
A:itonio Ferreira de Almeida Junior0) saneamento pela cducação-São Paulo, 1922-1 rol.-Doação.

Armando Arruda Sampaio-A profilaxia da tuberculose em São PauloSão Paulo, 1926-1 vol.-Doação.

Arnaldo Amado Ferreira-Contribuição ao estudo da drenagem em ginecologia-São Paulo, 1923-1 vol.-Doação.

Arthur Fajardo Filho-Contribuição a estudo higrienico do leite-São Paulo, 1927-1 vol.-Doação.

Benedicto Mendes de Castro-Contrihuição para o estudo das lesões do figado nos allienados-Sāo Paulo, 1926 -1 vol.-Doaşão.

Borba (Franoisco-) -El algodón-Buenos Aires, 1934-1 vol.- Permula.

Brazilio Rodrigues dos Santos-Da Escoliose e sua frequencia nos meninos das Escolas de São (P'aulo-São Paulo, 1923-1 vol-Doação.

Carlos Gomes de S. Thiago - Necessidade e obrigatoriedade de exames do aparelho visual dos empregados ferroviarios-São Paulo, 1927-1 vol.Doxação.

Cincinato Pomponet Filho-Coloração dias aguas de abastecimento publico de São Paulo.-São Paulo, 1928-1 vol.-Doação.

Felix Vianna Junior-Contribuigão ao estudo do leite e seu fornccimentio na cidade de São Paulo-São Paulo, 1921-1 vol.-Doação.

Fernandez, (Romelio J.-)-El extrato le tabaco en la chacra-Buenos Aires, 1934-1 vol.-Permuta.

Francisco Diciatteo-Da contaminação das masciuras de cloroformio-São Waulo, 1924-1 vol.-Doação.

fonorio Fabbri-O prblema higienico to Cancer-São Paulo -1 vol.-Doação.

Instrucciones para el uso del sulfuro de calcio $\rightarrow$ Buenos Aires, 1934-1 vol. -Permuta.

Israel Alves dos Santos-Um estudo epidemiologico-Sāo Paulo, 1925-1 vol.-Doação.

Ivo Lindemberg Quintanilha-Higiene prenatal-Sä Paulo, 192 $\underset{i}{\rightarrow}$ vol.-Doação.
Jeronymo La Terza-Dos pontadores de bacilos tifósos e paratifósos na Capital de São Paulo-Sāo Paulo, 1928-1 vol._Doaçä́.

João Areosa Oliveira de Mendonça Cortez-Contribuição ao estudo das Uricemias-São Paulo, 1924-1 vol.Doação.

João de Castro Simões-Da frequencia dos portadores sãos de bacilos de Klebs, Loeffler na cidade de $S$. Paulo—São IPlaulo, 1922-1 vol.-Doação.

José Daarte de Pateo Junior-Diagnostico precoce da lepra e seu valor sanitario-São Paulo, 1925-1 vol.Doaçálo.

José Reynaldo Marcondes-A reação de Gomes para o diagnostico precoce da lepra e seu valor profilatico-São Paulo, 1929-1 vol.-Doação.

Manoel de Toledo Passos-Profilaxia da ccgueira-São Paulo, 1928-1 vol. -Doação.

Mario da Costa Galvão-Inspeção sanitaria de Mogi das Cruzes—São Paulo, 1922-1 vol.- Doação.

Mario Pereira de Mesquita-Contribuição ao estudo da imunidade nas molestias infecciosas (papel da colesterina )-São (Paulo, 1931-1 vol.-Doação.

Max de Barros Erhart-Bacterias no dinheiro (papel moeda)-São Pauko, 1923-1 vol.—Doação.

Nestor de Barros Oliveira-Influrencia da rigidez cadaverica sobre o conteúdo dos ventriculos cardiacos-São Paulo, 1923-1 vol.-Doaşão.

Oswaldo Campos Barreto-Das conferencias $\mathrm{cm}$ medicina-São Paulo, 1927-1 vol.-Doação.

Piovano (Abelardo P.-)-Cultivo del tomate-Buenos Aires, 1934 1 vol.Permula.

Quirino Pucca-Sobre o ensino da medicina legal-São Paulo, 1926-1 vul. -Doação.

Raul Malheiros-Contribuição para 0 estudo epidemiologico das principais molestias infeciosar-São Paulo, 1926 -1 vol. -Doasão.

Reynaldo Porchat-Cattle Raising and the Meat Industry in Southern Bra- 
sil-Washinglun, 191\%-1 rol.-Doacão.

Salvador de Toledo Galrão-Incidencia e profilaxia da cisticenoose e do quisto hidatilo em São Paulo-Sắo Paulo, 1928-1 vol.-Dokação.

Samuel Barnsley Pessôa-Estudo dos componentes do aleo essencial de chenopodio, sua aplicação na profilaxia da Anquilostomose-(Trabalho do Instituto de Higiene)-São Pualo, 1922-1 vol.-Doação.

Samuel Leite Ribeiro-A fiscalização sanitaria dos generos alimenticios no Estado de São Paulo-São Paulo, 1926-1 voj-Doação.

Thomaz Bulgarelli-Ciontribuiçāo ao estudo de higiene pré-escolar-Sāo (Paulo, 1926-1 vol.—Doaçāo.

Ubaldino Antunes de Oliveira-Profilaxia social-São Paulo, 1924—1 vol. $\rightarrow$ Doação.

Vicente Pascarelli-Da proteção á primeira infancix em São Paulo-São Paulo, 1926-1 vol.-Doacão

Zoroastro de Oliveira Filho-Contribuição ao estudo da vacinação anti-diphterica preventoria pela anatoxina especifica-São Paulo, 1 vol.-Doaรูão.

\section{LITERATURA-(8)}

Anibal Freire da Fonseca-DiscursosRìo de Uanairo, 1924 - 1 vol.—Doasão.
Contos Sovieticos-Sāo Paulo, 1934-1 Vul.-Darção.

Durtain (Lue-)-Umilgens do Brasil e do Pampa-Rio de Janeiro, 1934-1 vol.-Doação.

Euripes Pedra-Tragedia em 4 atosLa Plata, $1923-1$ vol.-Permuta

\section{FISTORIA-(9)}

Historia, Geografia Politica, Viagens. Biografia, Heraldica, etc.

Carlos de Almeida Braga-A seçãn historica do Museu Paulista-São Paulo, 1933-1 vol.—Doação.

Ercole (Francisco-) - Da Carlo XIII a Carlo V-Firenze, 1932-1 vol.Compra.

Henrique A. Santa Rosa-Historia do Rio Amazonas-Pará, 1926-1 volDoação.

René Fulop-Miller-Lenine e Gandhi -Porto Alegre, .1934 - 1 vol.-Doaçāo.

Roth (Cecil-) -L'Ultima Republica Fiorentina-Firenze, 1929-1 vol.Compra.

Teschaver (Carlos-)-Historia do Rio Grande do Sul dos dois primeiros seculas.-Ponto Alegre, 1918-3 inls. -Permuta. 